\title{
Eicosapentaenoic Acid for Diabetic Abducens Nerve Palsy
}

\author{
Hidekatsu Yanai $^{\mathrm{a}, \mathrm{b}}$, Mariko Hakoshima ${ }^{\mathrm{a}}$
}

\section{To the Editor}

Ophthalmoplegia is a rare entity associated mainly with type 2 diabetes, and the overall prevalence of ophthalmoplegia cases was $0.32 \%$, further distributed into approximately $50 \%$ for abducens nerve palsy [1]. The cause and treatment for diabetic abducens nerve palsy remain largely unknown due to a rare entity. We experienced a diabetic old woman whose diabetic abducens nerve palsy was promptly improved by using eicosapentaenoic acid (EPA).

Our case was a 76-year-old woman with type 2 diabetes, hypertension and hypercholesterolemia, and her duration of diabetes was about 10 years. She has been treated by using sitagliptin $(50 \mathrm{mg} /$ day), insulin glargine (18 U/day), olmesartan (20 mg/day), benidipine hydrochloride ( $8 \mathrm{mg} /$ day) and pravastatin $(5 \mathrm{mg} /$ day). In July 2016, she felt a slight pain around right eye and developed diplopia and was admitted to our hospital in August 2016. On admission, her body mass index was $27.0 \mathrm{~kg} / \mathrm{m}^{2}$, and fasting plasma glucose, hemoglobin A1c (HbA1c), triglyceride, low-density lipoprotein (LDL)-cholesterol and high-density lipoprotein (HDL)-cholesterol were 288 $\mathrm{mg} / \mathrm{dL}, 9.4 \%, 267 \mathrm{mg} / \mathrm{dL}, 148 \mathrm{mg} / \mathrm{dL}$ and $41 \mathrm{mg} / \mathrm{dL}$, respectively. Brain magnetic resonance imaging did not show any abnormalities. We gave her a dietary therapy (1,200 kcal/day) and increased dairy dose of insulin glargine for the treatment of diabetes, and started to use EPA $(2,700 \mathrm{mg} /$ day $)$ to treat hypertriglyceridemia and hypo-HDL-cholesterolemia.

Her abduction impairment on admission was shown in Figure 1. Her diplopia and abduction impairment of right eye were promptly improved after the start of treatment using EPA (Fig. 1), and completely disappeared at 8 weeks after the start of EPA.

Although the cause of diabetic abducens nerve palsy has been unknown, atherosclerotic risk factors such as diabetes, hypertension, hyperlipidemia and hyperhomocysteinemia, and thrombosis have been reported to be associated with the development of diabetic abducens nerve palsy [2-5]. Pain which

Manuscript submitted July 7, 2017, accepted July 25, 2017

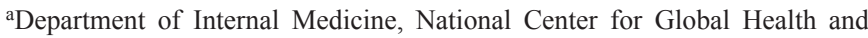
Medicine Kohnodai Hospital, Chiba, Japan

${ }^{\mathrm{b}}$ Corresponding Author: Hidekatsu Yanai, Department of Internal Medicine, National Center for Global Health and Medicine Kohnodai Hospital, 1-7-1 Kohnodai, Ichikawa, Chiba 272-8516, Japan.

Email: dyanai@hospk.ncgm.go.jp

doi: https://doi.org/10.14740/jem434w was also observed in our patient, has been suggested to be a common feature of microvascular ischemic ocular motor cranial nerve palsies [6], supporting a significance of atherosclerosis and thrombosis for the pathogenesis of diabetic abducens nerve palsy.

The treatment for diabetic abducens nerve palsy has been also largely unknown. Do et al reported that an acupuncture was effective to treat isolated abducens nerve palsy in a 58-year-old man with diabetes [7]. In their case report, the patient received a temporary prism for symptomatic relief; however, the improvement was not obtained after 2 months [7]. He received four acupuncture treatments over 11 weeks, and finally obtained complete resolution of diplopia [7].

In our case, diplopia and abduction impairment completely disappeared at 8 weeks after the start of EPA. EPA reduces serum triglyceride, increases serum HDL, and has anti-platelets and anti-inflammatory effects, improves endothelial function and blood rheology, and stabilizes atherosclerotic plaques [8]. Such anti-atherosclerotic and anti-thrombotic properties of EPA may be effective to treat diabetic abducens nerve palsy.

\section{Conflicts of Interest}

The authors declare that they have no conflicts of interest concerning this article.

\section{References}

1. Al Kahtani ES, Khandekar R, Al-Rubeaan K, Youssef AM, Ibrahim HM, Al-Sharqawi AH. Assessment of the prevalence and risk factors of ophthalmoplegia among diabetic patients in a large national diabetes registry cohort. BMC Ophthalmol. 2016;16:118.

2. Jung JS, Kim DH. Risk factors and prognosis of isolated ischemic third, fourth, or sixth cranial nerve palsies in the Korean population. J Neuroophthalmol. 2015;35(1):3740.

3. Sachdeva V, Mittal V, Pathengay A, Kekunnaya R, Gupta A, Rao BV. Isolated abducens nerve palsy with hyperhomocysteinemia: association and outcomes. Indian J Ophthalmol. 2013;61(10):598-600.

4. Mittal SO, Siddiqui J, Katirji B. Abducens nerve palsy due to inferior petrosal sinus thrombosis. J Clin Neurosci. 2017;40:69-71.

5. Kamouchi M, Wakugawa Y, Okada Y, Kishikawa K, Matsuo R, Toyoda K, Yasumori K, et al. Venous infarction 
$\mathbf{a}$
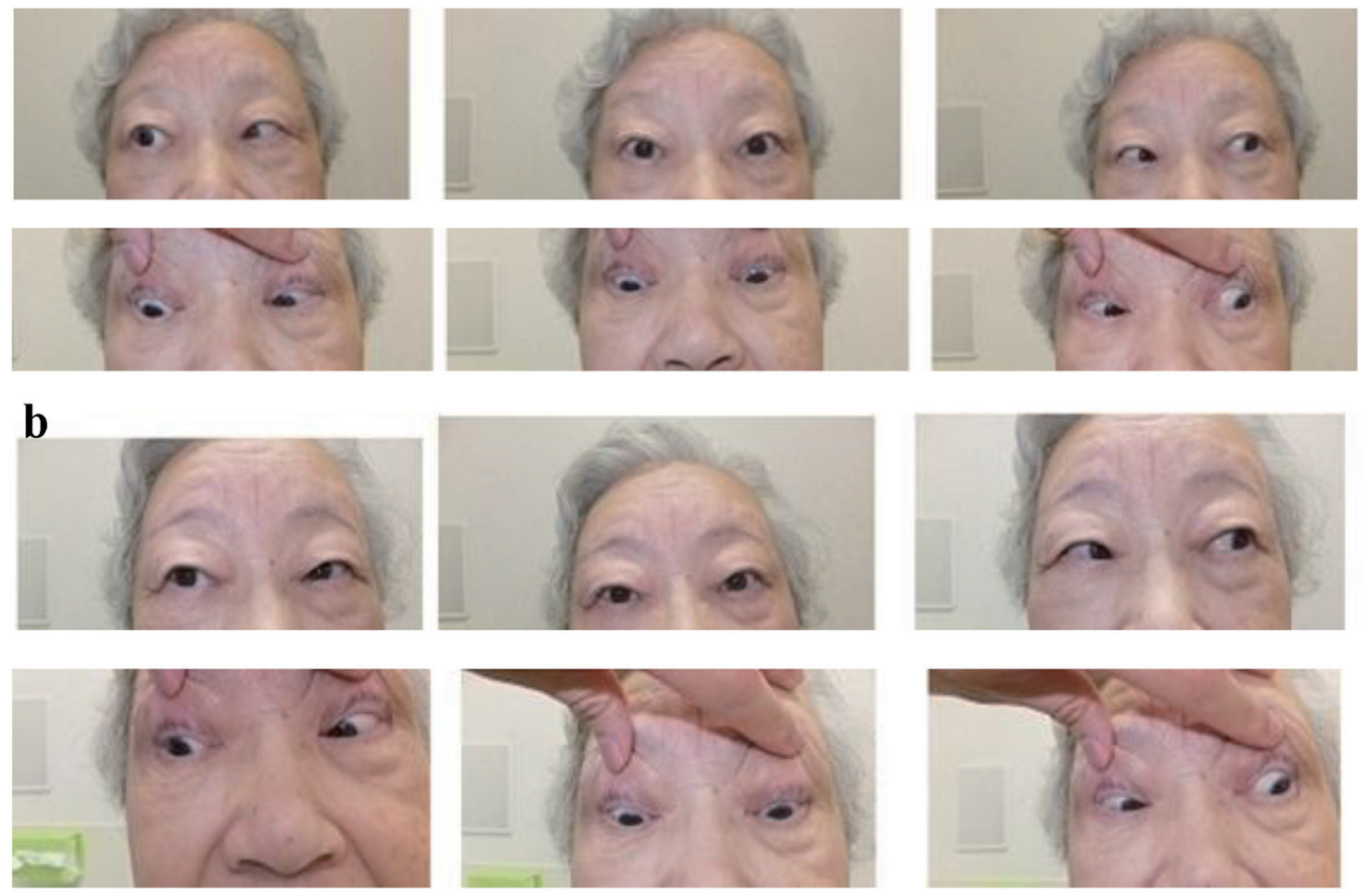

Figure 1. Our patient's abduction impairment on admission (a) and at 4 weeks after the start of treatment using EPA (b).

secondary to septic cavernous sinus thrombosis. Intern Med. 2006;45(1):25-27.

6. Wilker SC, Rucker JC, Newman NJ, Biousse V, Tomsak RL. Pain in ischaemic ocular motor cranial nerve palsies. Br J Ophthalmol. 2009;93(12):1657-1659.
7. Do A, Wahner-Roedler DL, Bauer BA. Acupuncture treatment of diplopia associated with abducens palsy: a case report. Glob Adv Health Med. 2014;3(4):32-34.

8. Yanai H, Yoshida H. Eicosapentaenoic Acids (EPA) for atherosclerotic diseases. J JSMUFF. 2014;8(3):215-220. 